\title{
Desarrollo Psicomotor en Niños de Nivel Socioeconómico Medio-Alto
}

\author{
LUISA SCHONHAUT B. ${ }^{1}$, MARIANNE SCHÖNSTEDT G. ${ }^{\text {la }}$, JORGE ÁLVAREZ L. ${ }^{1}$, \\ PATRICIA SALINAS A. ${ }^{1}$, IVÁN ARMIJO R. ${ }^{2 b}$ \\ 1. Facultad de Medicina Clínica Alemana - Universidad del Desarrollo. \\ 2. Escuela Psicología Universidad del Desarrollo. \\ a. Terapeuta. \\ b. Psicólogo.
}

\begin{abstract}
Psychomotor Development Among Children of Upper-Middle Class in Chile

Objective: Describe the psychomotor development (PMD) in upper middle class children, utilizing Bayley's Scale of Intellectual Development (BSID), internationally standarized. Patients and Methods: A total of 119 children in groups of 8,18 and 30 months were enrolled at the well-baby clinic in a Private Hospital in Santiago, Chile. BSID III was utilized in all cases. Children born preterm, or with neurological, genetic, or metabolic diseases were excluded. Results: BSID scores followed a normal distribution (100.3 \pm 10$)$. Prevalence of déficit was found to be $30 \%$ at 8 months, $7.7 \%$ at 18 months, and $2.7 \%$ at 30 months, without gender difference. Gross motor development and language predominated at 8 months. Ther threee subscales showed an upward trend, cognitive and motor scales most significantly. Gross motor scales, which had been deficient at 8 months became normal at 18 months. Conclusions: The high frequency of motor deficits found at 8 months of age was striking. While all scales improved, cognitive and motor scales were most significant. The effect of stimulation and upbringing patterns upon chil development is discussed, being important for future studies of causality.

(Key words: Psychomotor development, Bayley's Scale of Intellectual Development, screening). Rev Chil Pediatr 2010; 81 (2): 123-128
\end{abstract}

\section{RESUMEN}

Objetivo: Describir los puntajes de desarrollo psicomotor (DSM) en niños sanos de nivel socio económico medio alto mediante la Escala de Bayley de Desarrollo Infantil (BSID), estandarizada internacionalmente. Pacientes y Métodos: Estudio transversal descriptivo. Se enrolaron 119 niños 8, 18 y 30 meses, que acudie-

Trabajo recibido el 02 de octubre de 2009, devuelto para corregir el 24 de diciembre de 2009, segunda versión el 04 de enero 2010, aceptado para publicación el 23 de enero de 2010.

*Proyecto aprobado al comité de Ética de la Clínica Alemana-Universidad del Desarrollo.

*Estudio financiado por el proyecto SOCHIPE 2008013 en asociación con el proyecto FONIS SA7i20043.

* Los resultados de este trabajo fueron presentados en forma parcial en el XLIX Congreso Chileno de Pediatría y en XXVII Congreso de la Sociedad de Psiquiatría y Neurología de la Infancia y Adolescencia.

Correspondencia a:

Luisa Schonhaut Berman

E-mail: Ischonhaut@alemana.cl 
ron a control sano en una Clínica privada de Santiago de Chile, para la aplicación de BSID III. Se excluyeron los nacidos de pre-término o con enfermedad neurológica, genética o metabólica conocida. Resultados: Los puntajes obtenidos en BSID siguieron una distribución normal (100,3 \pm 10$)$. La prevalencia de déficit en el DSM fue $30 \%$ a los 8 meses, $7,7 \%$ a los 18 y 2,7\% a los 30 meses, no hubo diferencia por sexo. A los 8 meses predominó el déficit motor grueso y posteriormente el lenguaje. Las tres subescalas mostraron una tendencia al alza, las áreas cognitiva y motriz siguieron una progresión significativa. La motricidad gruesa, que estaba en el rango inferior a los 8 meses, fue normal a los 18. Conclusiones: Destaca la alta frecuencia de déficit motor en la población evaluada, especialmente a la edad de 8 meses. Todas las subescalas mostraron una tendencia al alza, siendo ésta diferencia significativa en las áreas cognitiva y motora. Se discute el efecto de la estimulación y los patrones de crianza en el desempeño de los niños, siendo necesario planificar estudios prospectivos para conocer su relación de causalidad.

(Palabras clave: Desarrollo psicomotor, Escala de Bayley de Desarrollo Infantil, screening).

Rev Chil Pediatr 2010; 81 (2): 123-128

\section{Introducción}

El desarrollo psicomotor (DSM) es un proceso evolutivo, multidimensional e integral, mediante el cual el individuo va dominando progresivamente habilidades y respuestas cada vez más complejas.

Alteraciones del desarrollo neuromadurativo se relacionan con bajo rendimiento $\mathrm{y}$ deserción escolar, trastornos neurológicos, psiquiátricos, emocionales y conductuales, déficit en las habilidades sociales, problemas de aprendizaje, menores oportunidades laborales y morbilidad en la adultez ${ }^{1,2}$.

Si bien cada ser humano al nacer tiene un potencial de desarrollo determinado congénitamente, su expresión final es resultado de la interacción de la genética con estímulos recibidos desde el entorno familiar, social, y comunitario. Probablemente ese sea el motivo por el cual la intervención temprana tiene alto impacto en el pronóstico de los niños con déficit del desarrollo 3 .

Por esta razón la detección precoz es una actividad central en los controles de supervisión de salud y supone un desafío permanente en pediatría, especialmente al evaluar niños con factores de riesgo sociales o biológicos.

Uno de los instrumentos más utilizados a nivel mundial en la pesquisa y objetivación de los déficits de DSM es la Escala Bayley de Desarrollo Infantil (BSID), siendo además considerado como patrón de referencia en estudios de validación de otros instrumentos ${ }^{4}$.
A pesar que el BSID carece de validación nacional, en nuestro país se emplea en programas de seguimiento de prematuros y en estudios sobre DSM asociado a factores de riesgo específicos $^{5-7}$.No obstante, desconocemos la situación de desarrollo de niños sanos, sin factores de riesgo biológicos y socioeconómicos.

Este estudio se enmarca en el proyecto FONIS SA7i20043 y proyecto SOCHIPE $2008013^{8}$, de validación de un test autoadministrado para la evaluación del DSM a las edades de 8, 18 y 30 meses, de acuerdo a la recomendación de la Academia Americana de Pediatría9.

El objetivo es describir los puntajes de desarrollo según Escala de Bayley de Desarrollo Infantil, en niños sanos, de nivel socio económico medio alto. El contar con esta muestra nos permitirá tener un grupo de referencia nacional para la comparación de otros grupos con factores de riesgo específicos.

\section{Pacientes y Método}

El presente estudio tiene un diseño transversal descriptivo. El Universo está conformado por lactantes y preescolares nacidos de término de nivel socioeconómico medio alto, pertenecientes al quinto quintil de ingreso, de acuerdo a la encuesta CASEN $2006^{10}$, que se atienden en forma habitual en las consultas de pediatría ambulatoria de una Clínica Privada de la Región Metropolitana de Santiago de Chile. 
La muestra, de oportunidad, fue conformada por 3 grupos independientes y no aleatorios de lactantes y preescolares de 8, 18 y 30 meses, que se enrolaron voluntariamente, hasta completar una muestra cercana a 40 niños por cada grupo etáreo, entre los meses de mayo 2008 y junio 2009. Destaca que un porcentaje superior al $80 \%$ de los padres invitados al estudio accedió a participar.

Se excluyeron los niños con factores de riesgo biológico importante, como los que tenían un diagnóstico conocido de enfermedad neurológica, congénita o metabólica, los nacidos prematuros (menores de 37 semanas de edad gestacional), aquellos cuyo adulto responsable no tuviese como idioma nativo el español. Dado que la participación fue voluntaria, se excluyeron también todos aquellos casos que declinaron participar. Cada niño se incluyó sólo una vez en el estudio.

A los padres o cuidadores principales de los niños, se les solicitó firmar un Consentimiento Informado y completar un formulario con datos de identificación, información demográfica y de riesgo biológico. Posteriormente, se fijó una fecha para la aplicación de BSID en una consulta adaptada para este fin. El test fue aplicado por una Terapeuta Ocupacional acreditada para dicha evaluación.

Escala de Bayley-III de Desarrollo Infantil ${ }^{11}$ : Escala que evalúa el desarrollo en niños de 1 a 42 meses. La prueba consta de tres subescalas diferenciadas: cognitiva, comunicación (comprensiva y expresiva) y motora con los componentes de motricidad gruesa y fina.

De acuerdo al manual del BSID, los puntajes totales de cada subescala fueron llevados a puntajes estándar, donde la media es 100 puntos y 1 DS es 15 puntos. En el análisis por áreas específicas (cognitiva, lenguaje expresivo, lenguaje comprensivo, motricidad fina y gruesa) la media estándar es de 10 puntos \pm 2 . Se consideró rendimiento en riesgo si el niño evaluado tenía, al menos en un dominio, un puntaje $<-1$ DS y retraso cuando era $<-2$ DS.

Análisis estadístico: Se realizó un análisis descriptivo de puntajes segmentados por edad, utilizando estadísticas de tendencia central, simultáneamente se verificó si las variables tenían distribución normal mediante la prueba de Schapiro Wilk; dado los resultados, fue posible utilizar análisis paramétrico (ANOVA) y prueba de Bonferroni, para comparar los puntajes obtenidos. Se consideró significativo un valor de $\mathrm{p}<0,05$. Se utilizó el software estadístico SPSS versión 16.0.

\section{Resultados}

Se aplicó el BSID a 119 niños que cumplían los criterios de inclusión al estudio. La totalidad de ellos tenía padres con educación superior y provenía de familias pertenecientes al quinto quintil de ingreso.

La distribución de acuerdo al puntaje obtenido en el BSID III siguió una curva normal (Schapiro Wilk).

En la tabla 1 se puede observar la frecuencia de niños catalogados como déficit en su DSM según edad y sexo. Catorce niños $(11,76 \%)$ fueron catalogados con riesgo y tres $(2,5 \%)$ con retraso del DSM. La tasa de déficit fue significativamente mayor a los 8 meses que a los 18 y 30 meses de edad $(p=0,001)$ (tabla 1$)$. No hubo diferencia por sexo.

Al desglosar por área de desarrollo deficitario destaca que 12 de los 13 lactantes de 8 meses con déficit del DSM, tenían compromiso motor grueso. Sólo un lactante tuvo déficit en las tres áreas evaluadas. A los 18 y 30 meses la totalidad del déficit, fue en el área de lenguaje.

En el análisis de logro de los hitos del desarrollo motor grueso evaluados a la edad de 8 meses, destaca que $88 \%$ de los lactantes se sentaba en forma estable, la mitad giraba de supino a prono, $40 \%$ se lograba poner en posición de gateo en cuatro puntos, y sólo $14 \%$ gateaba (figura 1).

En la tabla 2 y figura 2 se muestran la evolución de los puntajes obtenidos de acuerdo a la edad y área del desarrollo. La diferencia de medias, entre las edades, de acuerdo al test de ANOVA, fue significativa para las áreas motoras y cognitiva, pero no para el lenguaje. Los puntajes de motricidad se encontraban en el rango inferior a los 8 meses (promedio $87 \pm$ 12,9 ), para alcanzar la media a los 18 (promedio $101,7 \pm 13,4)$. 


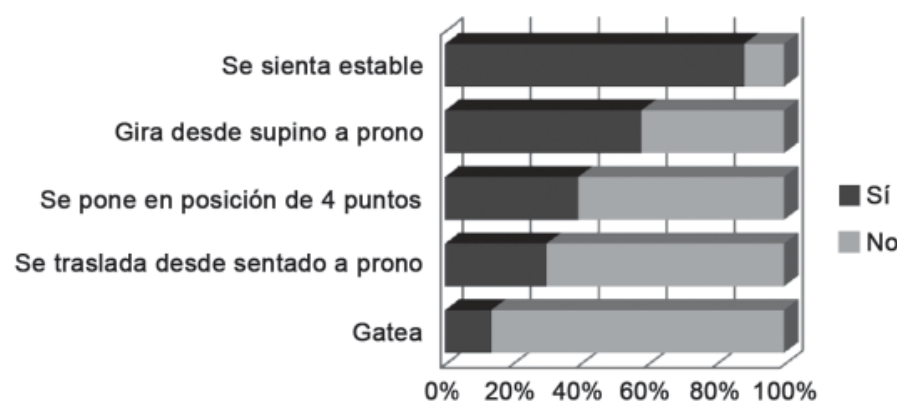

Figura 1. Porcentaje de logros de hitos de motricidad gruesa a los 8 meses.

Tabla 1. Frecuencia déficit del desarrollo psicomotor según Escala de Bayley

\begin{tabular}{|lcccc|}
\hline Muestra & & \multicolumn{2}{c}{$\begin{array}{c}\text { Escala de Bayley } \\
\mathbf{n}(\%) \\
\text { Riesgo }\end{array}$} & $\begin{array}{c}\text { Relación } \\
\mathbf{H} / \mathbf{M}^{*}\end{array}$ \\
\hline 8 metraso & \\
\hline 18 meses & 39 & $3(7,7 \%)$ & 0 & $7 / 6$ \\
\hline 30 meses & 37 & $1(2,7 \%)$ & 0 & $2 / 1$ \\
\hline Total & 119 & $14(11,8 \%)$ & $3(2,5 \%)$ & $1 / 0$ \\
\hline
\end{tabular}

*Relación H/M: relación entre hombres y mujeres.

Tabla 2. Comparación de puntajes según edad y subescala del Bayley

\begin{tabular}{|llccc|}
\hline Mes & & Cognitivo & Lenguaje & Motor \\
\hline 8 & Media & 97,44 & 99,65 & 87,07 \\
& DS & 8,2 & 12,1 & 12,9 \\
\multirow{2}{*}{18} & Media & 103,49 & 103,74 & 101,33 \\
& DS & 10,3 & 13,5 & 8,0 \\
\hline \multirow{2}{*}{30} & Media & 109,32 & 105,51 & 103,70 \\
& DS & 12,6 & 11,8 & 13,4 \\
\hline \multirow{2}{*}{ Significancia } & ANOVA & $\mathrm{p}<0,001^{*}$ & $\mathrm{p} 0,1$ & $\mathrm{p}<0,001^{*}$ \\
\hline
\end{tabular}

*Al aplicar la prueba de Bonferroni todas las combinaciones fueron estadísticamente significativas, excepto la comparación entre 8 y 18 meses.

\section{Discusión}

En nuestro estudio los puntajes de DSM, de acuerdo al BSID III, fueron en aumento desde los 8 a los 30 meses de edad. La distribución de los puntajes obtenidos siguió una Curva Normal, concordante con lo que ha sido publicado internacionalmente.

En nuestra muestra encontramos $14,3 \%$ de puntajes bajos, de ellos $11,8 \%$ en zona de riesgo y $2,5 \%$ en zona de déficit. Esta prevalencia es comparable a lo reportado tanto a nivel nacional como internacionalmente ${ }^{12-14}$.

El análisis desglosado por áreas reveló que la motricidad gruesa se encontraba descendida en casi un tercio de los lactantes de 8 meses, mientras que a los 18 meses se encontraba dentro del rango normal inferior, para continuar progresando hacia los 30. Las áreas de de lenguaje y cognitiva se mantuvieron siempre en rango promedio, pero con tendencia al alza, más destacado en el desarrollo cognitivo.

El presente estudio nos permite plantear hipótesis en relación a la progresión del DSM, especialmente llamativa en cuanto a la motricidad gruesa. La distribución de los puntajes obtenidos podría corresponder a una variante normal del

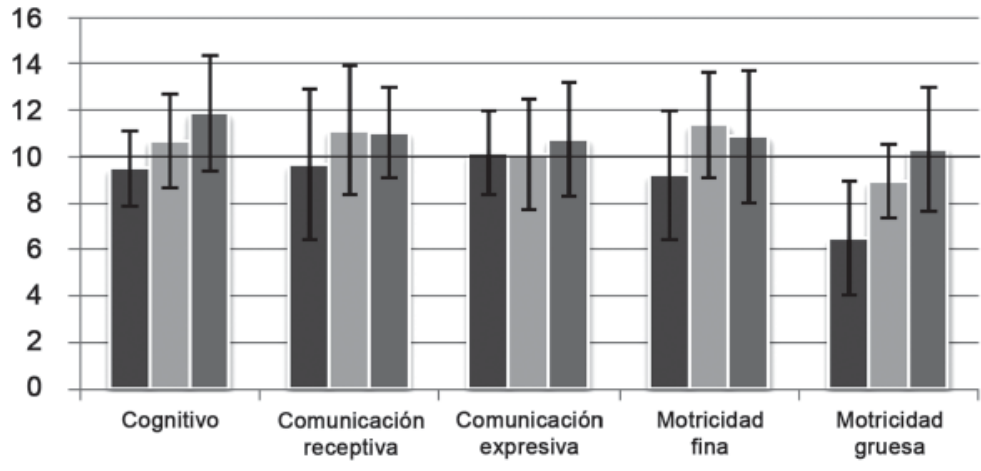

8 meses

[18 meses

20 meses
Figura 2. Comparación de los puntajes obtenidos en las distintas subescalas del Bayley. 
desarrollo, o bien a un trastorno transitorio y aislado, entidad a la que Illingworth clasificó como "disociaciones del desarrollo"15.

Según un estudio multicéntrico conducido por la OMS, tanto el crecimiento como el desarrollo siguen patrones similares en niños que cumplen con las condiciones ambientales óptimas, independiente de factores étnicos y genéticos. En dicho estudio, se refiere que las ventanas de normal adquisición de hitos del desarrollo motor grueso son tan amplias, que incluyen desde el percentil 1 al 99, atribuyendo las diferencias encontradas a patrones de crianza $^{16}$.

El impacto que diversos de patrones de crianza, como la posición al dormir o uso de implementos de bebé (como sillas nido, andador fijo o móvil, saltarinas) puedan tener en el desarrollo psicomotor ${ }^{17}$, no ha podido ser completamente demostrado. Dilucidar la relación que pueda existir entre las llamadas "disociaciones del desarrollo" y el uso de estos implementos o la posición al dormir, excede los objetivos del presente manuscrito. Distintos autores han podido establecer que, posterior al comienzo de la campaña "Back to Sleep" de la Academia Americana de Pediatría para prevenir la muerte súbita ${ }^{18}$, los lactantes comenzaron a tener una menor experiencia en posición prona y este hecho se asoció a un aumento en la prevalencia de retraso del desarrollo de la motricidad gruesa a edades precoces, con normalización posterior ${ }^{19-21}$.

Con toda la información acumulada hasta la fecha sabemos que dormir de espalda (supino) es una manera efectiva de prevenir Muerte Súbita y que debe seguir siendo una recomendación fundamental. No obstante lo anterior, a la luz de nuestras observaciones y lo reportado por otros autores ${ }^{18-21}$, tenemos buenos argumentos para sugerir a los padres aumentar el tiempo de actividad supervisada en prono durante el día ("tummy time") 22 , ya que esta práctica podría tener un impacto beneficioso en la evolución de la motricidad de los niños.

Este estudio nos permite conocer la situación de desarrollo de niños sanos, sin factores de riesgo biológicos y aventajados socioeconómicamente, pudiendo ser usado como referencia para el análisis del comportamiento de desarrollo de otros grupos a nivel nacional. No obstante, para el correcto análisis de los resultados, debemos considerar las limitaciones del estudio, que corresponde a la comparación de los puntajes del DSM tres submuestras independientes de niños pertenecientes a tres grupos etáreos, reclutados en forma voluntaria y transversal, debiendo planificar un diseño prospectivo para poder responder efectivamente a las interrogantes planteadas.

\section{Conclusión}

La distribución de los puntajes obtenidos siguió una Curva Normal, concordante con lo que ha sido publicado internacionalmente. Se evidenció una frecuencia de $14,3 \%$ de puntajes bajos. El análisis desglosado por áreas, reveló que un $28 \%$ de los lactantes presentó un puntaje bajo en motricidad gruesa a los 8 meses, y que esta área del desarrollo se encontraba dentro del rango normal en los niños de mayor edad. Se plantea la posible influencia de patrones de crianza en la adquisición de hitos del DSM, especialmente la motricidad gruesa, siendo necesario planificar estudios prospectivos para establecer la relación de causalidad.

\section{Referencias}

1.- Manterola A, Avendaño A, et al: Examen neurológico y rendimiento escolar: correlaciones a siete años Plazo. Rev Chil Pediatr 1989; 60 (3): 157-65.

2.- Illingworth R: Desarrollo del niño. Editorial Churchill Livingstone novena edición, España 1992.

3.- GuralnickMJ: The Effectiveness of Early Intervention. Baltimore, MD: Paul H. Brookes Publishing Co; 1997.

4.- Yu LM, Hey E, Doyle LW, et al, Magpie Trial FollowUp Study Collaborative Group: Evaluation of the Ages and Stages Questionnaires in identifying children with neurosensory disability in the Magpie Trial follow-up study. Acta Paediatr 2007; 96 (12): 1803-8.

5.- Casrillo-Durán C, Perales C, Hertrampf E, Marín V, Icaza $G$ : Effect of zinc supplementation on development and growth of Chilean infants. Journal of Pediatrics 2001; 138 (2): 229-35.

6.- Vega, et al: Chronic exposure to environmental lead in Chilean infants. II: Effects on the psychomotor development. Rev Med Chile 1999; 127 (1): 28-37. 
7.- De Andraca I, Pino P, de La Parra A, Rivera F, Castillo $M$ : Factores de riesgo para el desarrollo psicomotor en lactantes nacidos en óptimas condiciones biológicas. Rev Saúde Pública 1998; 32 (2): 138-47.

8.- Schonhaut L, Salinas P, Armijo I, Schonstedt M, Álvarez $J$, Manríquez M: Screening de desarrollo psicomotor en la consulta pediátrica: evaluación del una prueba de auto-reporte de los padres. Rev Chil Pediatr 2009; 80 (6): 513-9.

9.- Council on Children with Disabilities, Section on Developmental Behavioral Pediatrics, Bright Futures Steering Committee and Medical Home Initiatives for Children with Special Needs Project Advisory Committee: Identifying Infants and Young Children with Developmental Disorders in the Medical Home: An Algorithm for Developmental Surveillance and Screening. Pediatrics 2006; 118: 405-20.

10.- www.mideplan.cl

11.- Bayley N: Bayley Scales of Infant and Toddler Development. Socio-Emotional Scale. Third Edition. Psych Corp. 2005.

12.- Subsecretaría de Salud Pública División de Planificación Sanitaria: II Encuesta de Calidad de Vida y Salud, Chile 2006. Disponible en: http://epi.minsal.cl/epi/html/ sdesalud/calidaddevida2006/Informe $\% 20$ Final $\% 20$ Encuesta $\% 20$ de $\% 20$ Calidad $\% 20$ de $\% 20$ Vida $\% 20$ y $\% 20$ Salud\%202006.pdf

13.- Committee of Children with Disabilities: Developmental surveillance and screening of infant and young children. Pediatrics 2001; 108 (1): 192-5.
14.- Boyle CA, Decoufle P, Yeargin-Allsoop MY: Prevalence and health impact of developmental disabilities. Pediatrics 1994; 93: 863-5.

15.- Illingworth R: Dissociation as a guide to developmental assessment. Arch Dis Childhood 1958; 33: 118-22.

16.- Who Multicentre Growth Reference Study Group: WHO Motor Development Study: Windows of achievement for six gross motor development milestones. Acta Paediatr 2006; Suppl 450: 86-95.

17.- Pin T, Eldridge B, Galea M: A review of the effects of sleep position, play position, and equipment use on motor development in infants. Developmental Medicine \& Child Neurology 2007; 49: 858-67.

18.- American Academy of Pediatrics, Task Force on Infant Positioning and Sids: Positioning and SIDS. Pediatrics 1992; 89: 1120-6.

19.- Hunt L, Fleming P, Golding J, The Alspac Study Team: Does the sleeping position have any adverse effects on the child? I. Health in the first 6 months. Pediatrics 1997; 100: 1-9.

20.- Dewey C, Fleming P, Golding J, and The Alspac Study Team: Does the Supine Sleeping Position Have Any Adverse Effects on the Child? II. Development in the First 18 Months Pediatrics 1998; 101 (1): 1-5.

21.- Davis B, Moon R, Sachs HY, Ottolini M: Effects of Sleep Position on Infant Motor Development. Pediatrics 1998; 102 (5): 1135-40.

22.- Carmeli E, Marmur R, Cohen AY, Tirosh E: Preferred sleep position and gross motor achievement in early infancy. Eur J Pediatr 2009; 168: 711-5. 